\title{
Lumbar myofascial flap for pseudomeningocele repair
}

\author{
Sanjay N. Misra, M.D., Howard W. Morgan, M.D., and Ross Sedler, M.D. \\ Department of Neurosurgery, Denver Health Medical Center, University of Colorado Health Science \\ Center, Denver, Colorado; Department of Neurosurgery, University of Texas Southwestern, Medical \\ Center at Dallas; and Division of Neurosurgery, Texas Technical University, Lubbock, Texas
}

\begin{abstract}
Object. Initial management for lumbar pseudomeningoceles entails the closed external drainage of cerebrospinal fluid (CSF) with or without blood patch application. The presence of longstanding pseudomeningoceles and those associated with nonmicroscopic dural tears can be more problematic. Additionally the failure of nonoperative measures may necessitate surgery. Ideally the procedure should involve repairing the dural defect, removing the encapsulated cavity of the pseudomeningocele, and obliterating the extraspinal dead space to minimize the recurrence of the problem.

Methods. The authors describe a technique performed in 12 patients with large $(>5$-cm-diameter) pseudomeningoceles referred for management following the failure of less aggressive measures. Diagnosis was based on symptoms of lumbar wound swelling, postural headaches, back and leg pain, and was confirmed by imaging studies. In all patients subarachnoid CSF drainage and initial operative attempts to obliterate the pseudomeningocele had failed. They were treated between July 1990 and July 1998. The cause of the pseudomeningoceles was lumbar discectomy (four patients), lumbar decompression (one patient), lumbar decompression and placement of instrumentation (five patients), and intradural procedures (two patients). Their mean age was 47.9 years (range 20-67 years), and they presented at a mean of 5.5 months postoperatively (range 3 weeks -37 months). In all cases there was a satisfactory repair of the pseudomeningocele, dead space obliteration, and long-term symptomatic resolution.

Conclusions. Lumbar myofascial advancement for this problem is a useful technique in cases of symptomatic pseudomeningoceles. This technique requires the medial advancement of the musculofascial units of the paravertebral muscles for a layered closure over the exposed spinal canal with obliteration of the pseudomeningocele.
\end{abstract}

\section{KEY WoRDS • pseudomeningocele • cerebrospinal fluid leak • durotomy • lumbar spine}

The incidence of intraoperative durotomy ranges from 3.1 to $14 \% .4,11,21,23$ It is greater for reoperations $(17.4 \%)$ than the initial surgery $(1.8 \%) .{ }^{21}$ In the majority of cases these are recognized and repaired primarily. They can be reinforced with fibrin glue and the patient should then undergo a period of bed rest. Some authors have argued that it is the primary repair and not bed rest that is critical to the success of this treatment. ${ }^{6,9}$ Despite meticulous repair, a postoperative lumbar pseudomeningocele may occur. Pseudomeningoceles warrant immediate attention because their associated morbidity ranges from headache and lumbar discomfort to severe back pain and radiculopathy secondary to nerve rootlet entrapment. ${ }^{1,3,8,14}$ Should the pseudomeningocele result in a cutaneous CSF leak, the patient is exposed to the risk of central nervous system infection. ${ }^{12}$ The incidence of lumbar pseudomeningoceles is reported to be between 0.3 and 5\%., ${ }^{4,922,23}$ Surgical repair is indicated if the CSF drainage, which may be supplemented by epidural blood patch application, does not result in a resolution of the problem. The failure of nonoperative treatment and simple surgery by using dural repair with subsequent reaccumulation of an encapsulated pseu-

Abbreviations used in this paper: $\mathrm{CSF}=$ cerebrospinal fluid; $\mathrm{MR}=$ magnetic resonance. domeningocele requires more aggressive treatment. When posterior spinal instrumentation and paravertebral muscle atrophy are present, the situation is exacerbated because there is much less of a tamponade effect acting upon a dural CSF leak.

\section{CLINICAL MATERIAL AND METHODS}

\section{Patient Population}

Between 1990 and 1998 we treated 12 patients referred for the management of postoperative pseudomeningoceles larger than $5 \mathrm{~cm}$ in diameter. There were eight women and four men whose mean age was 47.9 years (range 20-67 years). Table 1 provides a summary of the primary diagnoses, which required treatment and were complicated by the pseudomeningocele formation. In 10 of 12 cases pseudomeningocele occurred as a complication of extradural surgery, and five were procedures involving placement of posteri-or spinal instrumentation with pedicle screws. In two of 12 cases the lesion formed after intradural procedures. In three patients a previous surgery to correct the pseudomeningocele at the referring hospital had failed. The mean interval between the prior lumbar operation and referral with pseudomeningocele with failed nonoperative treatment was 5.5 months (range 3 weeks-37 months). 
TABLE 1

Causes of lumbar pseudomeningoceles in 12 patients

\begin{tabular}{lc}
\hline \multicolumn{1}{c}{ Cause } & No. of Cases \\
\hline lumbar discectomy & 4 \\
lumbar stenosis decompression & 1 \\
lumbar degeneration requiring decompression & \\
$\quad$ \& instrumentation & 5 \\
intradural tumor (neurilemmoma) resection & 1 \\
tethered spinal cord release & 1 \\
\hline
\end{tabular}

Ten patients suffered chronic postoperative back discomfort associated with a ballotable subcutaneous swelling consistent with a fluid collection; nine complained of severe postural headaches and radicular pain, suggestive of nerve root herniation; and three complained of a persistent postoperative lumbar swelling as their only symptom. Eight of these symptomatic patients had a history of multiple prior lumbar operations (Table 2).

\section{Surgical Technique}

The concept of the myofascial pseudomeningocele repair reinforced by the myofascial advancement is illustrated in Fig. 1. The steps are as follows. 1) The wound is reopened with rostrocaudal extension, as indicated for appropriate exposure. The entire pseudomeningocele cavity is opened, and subcutaneous dissection is extended to obtain a wide exposure of thoracolumbar fascia. The pseudomeningocele space within the deep muscular planes is also laid open. Further bone removal is conducted as indicated to expose the dura and identify the site of dural leakage (Fig. 1A and B). 2) If a large defect is present, then a tensor fascia lata graft is harvested in the usual manner. 3) This step is performed using the microscope, which provides for both superior illumination and magnification. The dural defect is repaired either primarily or with the incorporation of a fascia lata graft. Prior to this, any herniated nerve rootlets are identified, returned to the intradural space, and protected during the dural repair. This is achieved using a microdissector to retain the rootlets within the dura as it is closed. 4) Subcutaneous fat is placed loosely over the site of the dural repair. 5) Subcutaneous lateral dissection immediately superficial to the thoracolumbar fascia is performed. Vertical bilateral parallel incisions are made in this fascia approximately $5 \mathrm{~cm}$ lateral to the midline. These incisions are brought two segments above the vertebral levels of the pseudomeningocele. Inferiorly these are carried to the level of the posterior iliac crest (Fig. 1C).

Using blunt finger dissection to the depth of the transverse processes, the erector spinae muscles are mobilized in a lateromedial direction (Fig 1D). This dissection results in a vascularized myofascial flap attached at the superior and inferior poles. The paraspinous muscles comprising the myofascial unit are reapproximated over this fat, obliterating the dead space. Care is taken during this step not to apply excess compression to the thecal sac with resultant compromise of intradural neural structures. The thoracolumbar fascia, comprising the fascial component of the flap, is closed, however, in a watertight fashion. In-
TABLE 2

Number of lumbar procedures prior to referral for pseudomeningocele repair

\begin{tabular}{cc}
\hline \hline No. of Ops & No. of Cases \\
\hline 1 & 4 \\
2 & 5 \\
3 & 2 \\
4 & 1 \\
\hline
\end{tabular}

terrupted sutures are placed methodically at 5- to $10-\mathrm{mm}$ intervals and then reinforced using an uninterrupted suture (Fig. 1E). The lateral thoracolumbar fascial relaxing incisions are left open. 6) The subcutaneous fascia is tacked down to the thoracolumbar region by using the interrupted fascia. Subcutaneous and cutaneous closure is conducted in layers in the standard fashion. A lumbar drain is placed through a site separate from the surgical wound (either above or lateral to it).

This six-step technique was performed in all 12 cases. Muscle groups that comprise the myofascial flap are the erector spinae arising from the iliac crest and lumbar vertebral spinous processes (with their insertion being the posterior aspects of the ribs). Care is taken to preserve the lateral aspects of the paraspinous muscle flaps, because this is the site of the segmental lateral perforating arteries supplying the muscle and the overlying fascia, which comprise the functional flap. Because medial perforating arteries were divided at the time of the initial operation, the flap's viability rests on these lateral perforating vessels. Both the superior and inferior attachments of the paraspinous muscles and fascia are preserved.

\section{RESULTS}

In all cases there was successful closure of the pseudomeningocele. No recurrences were demonstrated on follow-up imaging at 2 to 4 months postoperatively. Symptoms of postural headaches and radicular pain referable to the pseudomeningocele preoperatively were resolved in eight of the nine patients. The three patients with a lumbar subcutaneous swelling but without postural headaches or symptoms of nerve root entrapment remained asymptomatic postoperatively. There were no procedure-related complications.

\section{ILLUSTRATIVE CASES}

Two cases are described to illustrate the indications for this technique in the management of postoperative lumbar pseudomeningoceles.

\section{Case 1}

This 65-year-old woman underwent lumbar decompression and instrumentation-augmented fusion for degenerative disease. She was referred for further management because of ongoing postoperative lumbar and radicular pain. Local pressure on the healed wound exacerbated her radicular symptoms. This was strongly suggestive of nerve rootlet entrapment in the area of the dural defect. (Fig. 2 

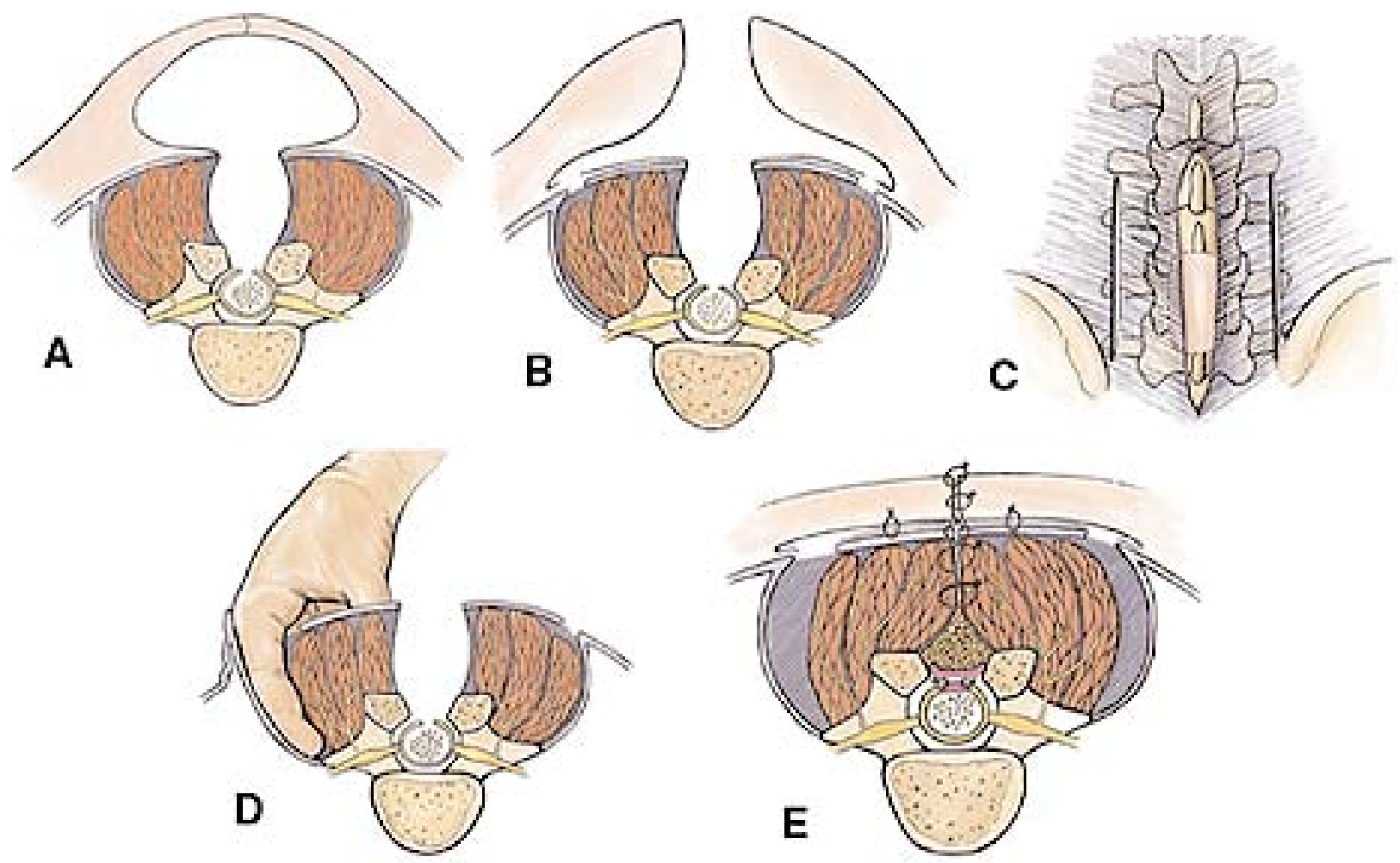

Fig. 1. Illustration depicting the individual steps involved in carrying out a myofascial advancement for pseudomeningocele obliteration. A: Axial view of the pseudomeningocele. B: Axial view demonstrating the identification of the pseudomeningocele and the lateral fascial incisions. C: Longitudinal view of the lateral fascial incisions extending to the posterior iliac crest. D: Lateral finger dissection mobilizing the myofascial flap. E: Dural repair with overlying fat graft and multilayered wound closure.

left and center). Prior to referral she had undergone a prolonged period of bed rest and closed lumbar subarachnoid CSF drainage after a lumbar pseudomeningocele was diagnosed. There had been partial relief of symptoms during that time; however, symptoms returned upon cessation of CSF drainage. Intraoperatively a dural defect causing rootlet herniation was identified (Fig. 2 right). The rootlet herniation was reduced, the dura repaired, and the pseu- domeningocele cavity obliterated using the aforedescribed technique (Fig. 3). Postoperatively the patient was managed with bed rest for 72 hours.

\section{Case 2}

This 60-year-old woman had undergone a unilateral hemilaminotomy and discectomy. The postoperative peri-
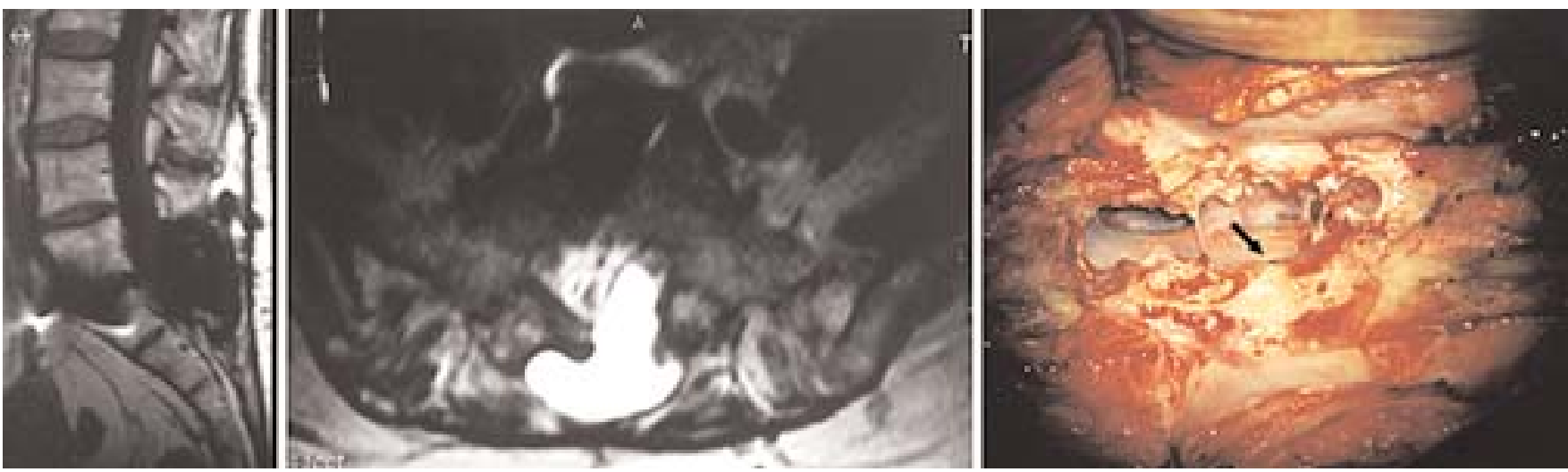

Fig. 2. Case 1. Left: Sagittal lumbar MR image demonstrating a pseudomeningocele contiguous with the thecal sac. Center: Axial MR image revealing the pseudomeningocele's communication with and displacement of the thecal sac. Right: Intraoperative photograph showing the interior of the pseudomeningocele after reopening of the prior lumbar wound. The pinpoint dural defect is seen communicating with the subarachnoid space. 

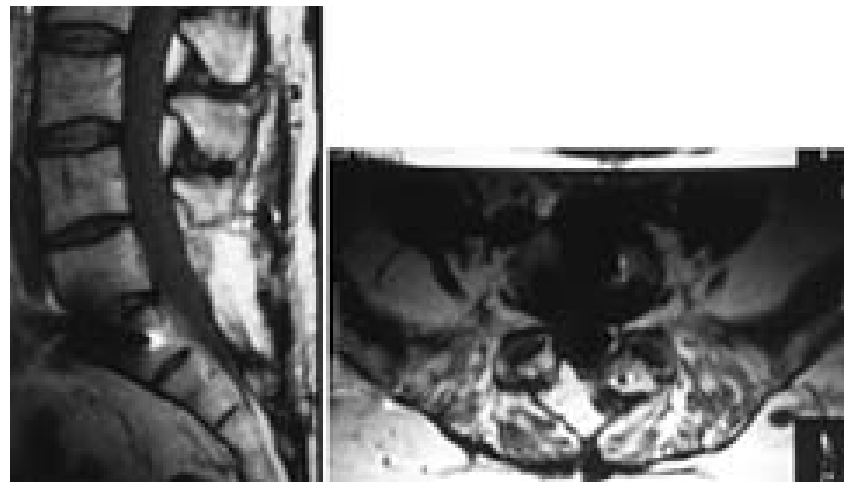

Fig. 3. Case 1. Postoperative MR images. Left: Sagittal image obtained at 3 months demonstrating the satisfactory obliteration of the pseudomeningocele. Preoperative symptoms resolved. Right: Axial image revealing extradural fat with the reinforcement provided by the myofascial repair in the midline.

od was uncomplicated; however, she developed ongoing postural headaches, accompanied by unilateral radicular pain when standing. A diagnosis of postoperative pseudomeningocele was confirmed on imaging studies (Fig. 4 upper). Axial MR imaging demonstrated extension of the pseudomeningocele into the subcutaneous plane through a dehiscence in the thoracolumbar fascia. Her symptoms persisted despite a period of bed rest and lumbar subarachnoid CSF drainage. She was referred for further management.

Because she had undergone a satisfactory trial of nonoperative treatment for this condition, which ultimately failed, pseudomeningocele repair was indicated. Operative exposure was increased by laminectomy at the level of the prior hemilaminotomy. This permitted identification of the site of the dural breach (Fig. 4 center), reduction of the rootlet herniation, and repair of this dural defect with a fascia graft (Fig. 4 lower). Postoperatively continuous lumbar CSF drainage was performed for 5 days to protect the site of the dural repair.

\section{DISCUSSION}

The aforedescribed technique of myofascial advancement is not recommended as the first option in the treatment of a postoperative lumbar pseudomeningocele. If the lesion forms immediately after surgery due to a repaired or unrecognized durotomy or following intradural procedures requiring durotomy, immediate subarachnoid CSF drainage is suggested. ${ }^{3,20,23}$ The presence of the subcutaneous fluctuant collection serves as an indication of the degree of response to this treatment. If the collection fails to diminish, a ball valve effect is indicated, with extradural accumulation. In this situation, computerized tomography-guided percutaneous aspiration with either extradural blood or fibrin glue patch application is a feasible option. $^{13,15}$

Should these measures fail and the pseudomeningocele becomes a symptom-producing encapsulated lesion, surgical exploration is indicated. Paravertebral muscle atrophy is a recognized consequence of lumbar procedures possibly related to damage to the posterior rami of the lumbar spinal nerves. ${ }^{2}$ This may be more likely in instrumentation-augmented cases because of the requisite lateral extent of spinal exposure and consequent posterior rami injury. ${ }^{2}$ As a consequence there is less available muscle bulk due to denervation atrophy. Thus, there is less soft tissue to tamponade the CSF leak. The resultant paravertebral dead space becomes filled with CSF and subsequently forms the pseudomeningocele cavity. Of note, eight of the 12 patients presented had undergone more than one lumbar operation in the past. The deficiency in the paravertebral muscles is in contrast to the intrinsic strength of the thoracolumbar fascia. This fascia has its
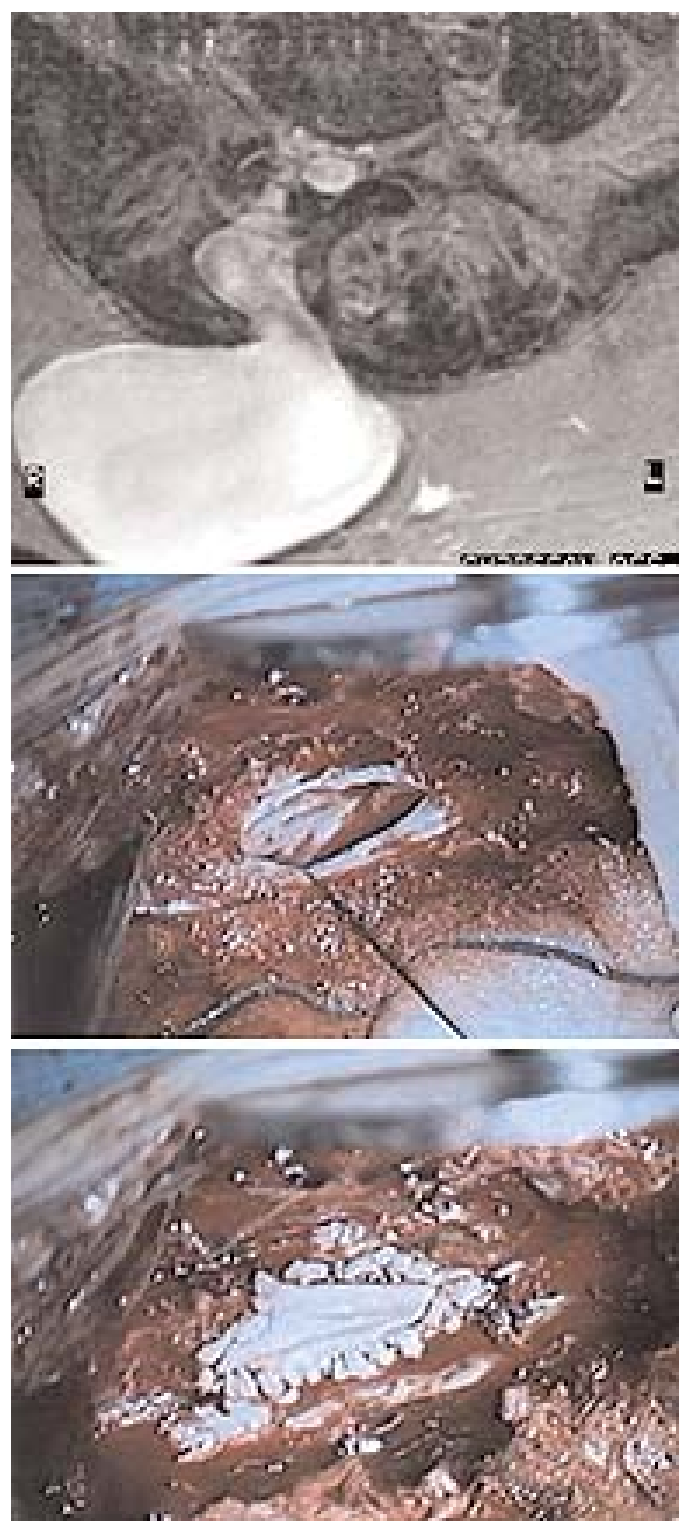

Fig. 4. Case 2. Upper: Axial lumbar MR image revealing a large pseudomeningocele extending into the subcutaneous plane. Center: Identification of the dural defect after extending the exposure. This was done by completion of the laminectomy at the level of the prior hemilaminotomy and discectomy. Lower: Repair of the dural defect with a fascia lata patch graft. 
origin in the eight to 12th ribs and the latissimus dorsi. It is reinforced by the fused aponeuroses of the external oblique, internal oblique, and transversus abdominis muscles. With its attachment inferiorly to the sacrum and iliac crest, it forms a strong band spanning the posterior lumbar region. Transposed with the underlying muscle and an intact vascular supply, ${ }^{24}$ it reinforces the tamponade effect of the extradural tissues on the site of dural weakness.

The utility of the lumbar paravertebral fascial, musculofascial, and fasciocutaneous flaps is not a new concept. It has a long-established role in the repair of myelomeningoceles in infants and lumbar soft-tissue defects. ${ }^{7,10,16-19,24}$ The same techniques have been used for repair of lumbar defects following soft-tissue loss with exposure of canal and placement of instrumentation. ${ }^{5}$ Integrating the experience from the other indications into the management of refractory postoperative lumbar pseudomeningoceles has met with success. Incidental durotomy or dural CSF leakage is an unforeseen but not unforeseeable event in lumbar surgery. Primary repair at the time is ideal and in the case of pseudomeningocele formation CSF drainage is a tried-and-true treatment modality. The refined technique described here is required when the aforementioned options fail.

\section{CONCLUSIONS}

Although the initial management for postoperative lumbar pseudomeningoceles is nonoperative, the aforedescribed technique has a place in the surgeon's armamentarium. It finds application in cases in which aggressive external CSF drainage and initial surgical attempts to stop the recurrence of the pseudomeningocele have failed. In these recalcitrant cases, this technique of methodical multilayered pseudomeningocele repair and obliteration has been shown to have favorable results.

\section{Acknowledgment}

We acknowledge the efforts of S. Truex in the preparation of the illustrations and the manuscript.

\section{References}

1. Aldrete JA, Ghaly R: Postlaminectomy pseudomeningocele. An unsuspected cause of low back pain. Reg Anesth 20:75-79, 1995

2. Boelderl A, Daniaux H, Kathrein A, et al: Danger of damaging the medial branches of the posterior rami of spinal nerves during a dorsomedian approach to the spine. Clin Anat 15:77-81, 2002

3. Bosacco SJ, Gardner MJ, Guille JT: Evaluation and treatment of dural tears in lumbar spine surgery: a review. Clin Orthop 389:238-247, 2001

4. Cammisa FP Jr, Girardi FP, Sangani PK, et al: Incidental durotomy in spine surgery. Spine 25:2663-2667, 2000

5. Dumanian GA, Ondra SL, Liu J, et al: Muscle flap salvage of spine wounds with soft tissue defects or infection. Spine 28: 1203-1211, 2003

6. Eismont FJ, Wiesel SW, Rothman RH: Treatment of dural tears associated with spinal surgery. J Bone Joint Surg Am 63: 1132-1136, 1981

7. Fiala TG, Buchman SR, Muraszko KM: Use of lumbar periosteal turnover flaps in myelomeningocele closure. Neurosurgery 39:522-526, 1996

8. Hadani M, Findler G, Knoler N, et al: Entrapped lumbar nerve root in pseudomeningocele after laminectomy: report of three cases. Neurosurgery 19:405-407, 1986

9. Hodges SD, Humphreys SC, Eck JC, et al: Management of incidental durotomy without mandatory bed rest. A retrospective review of 20 cases. Spine 24:2062-2064, 1999

10. Iacobucci JJ, Marks MW, Argenta LC: Anatomic studies and clinical experience with fasciocutaneous flap closure of large myelomeningoceles. Plast Reconstr Surg 97:1400-1410, 1996

11. Jones AA, Stambough JL, Balderston RA, et al: Long-term results of lumbar spine surgery complicated by unintended incidental durotomy. Spine 14:443-446, 1989

12. Koo J, Adamson R, Wagner FC Jr, et al: A new cause of chronic meningitis: infected lumbar pseudomeningocele. Am J Med 86:103-104, 1989

13. McCormack BM, Taylor SL, Heath S, et al: Pseudomeningocele/CSF fistula in a patient with lumbar spinal implants treated with epidural blood patch and a brief course of closed subarachnoid drainage. A case report. Spine 21:2273-2276, 1996

14. O'Connor D, Maskery N, Griffiths WE: Pseudomeningocele nerve root entrapment after lumbar discectomy. Spine 23: 1501-1502, 1998

15. Patel MR, Louie W, Rachlin J: Postoperative cerebrospinal fluid leaks of the lumbosacral spine: management with percutaneous fibrin glue. AJNR 17:495-500, 1996

16. Ramasastry SS, Cohen M: Soft tissue closure and plastic surgical aspects of large open myelomeningoceles. Neurosurg Clin N Am 6:279-291, 1995

17. Ramasastry SS, Schlechter B, Cohen M: Reconstruction of posterior trunk defects. Clin Plast Surg 22:167-185, 1995

18. Shevach I, Brodovski S, Rappaport ZH: Myelomeningocele repair by a combined fasciocutaneous flap closure. Technical note. Acta Neurochir 115:62-63, 1992

19. Shillito J, Matson DD: An Atlas of Pediatric Neurosurgical Operations. Philadelphia: WB Saunders, 1982, pp 474-479

20. Stambough JL, Templin CR, Collins J: Subarachnoid drainage of an established or chronic pseudomeningocele. J Spinal Disord 13:39-41, 2000

21. Stolke D, Sollmann WP, Seifert V: Intra- and postoperative complications in lumbar disc surgery. Spine 14:56-59, 1989

22. Teplick JG, Peyster RG, Teplick SK, et al: CT identification of postlaminectomy pseudomeningocele. AJR 140:1203-1206, 1983

23. Wang JC, Bohlman HH, Riew KD: Dural tears secondary to operations on the lumbar spine. Management and results after a two-year-minimum follow-up of eighty-eight patients. J Bone Joint Surg Am 80:1728-1732, 1998

24. Wilhelmi BJ, Snyder N, Colquhoun T, et al: Bipedicle paraspinous muscle flaps for spinal wound closure: an anatomic and clinical study. Plast Reconstr Surg 106:1305-1311, 2000

Manuscript received July 17, 2003.

Accepted in final form August 26, 2003.

Address reprint requests to: Sanjay N. Misra, M.D., Department of Neurosurgery, Denver Health Medical Center, University of Colorado Health Science Center, 777 Bannock Street, MC 0206, Denver, Colorado 80204. email: sanjay.misra@dhha.org. 